\title{
Structured matrix computations in non-Euclidean geometries: algorithms and applications
}

\section{Editorial of the special issue dedicated to a workshop held at CIRM, Luminy (France), October 8-12, 2012}

\author{
Peter Benner • Miloud Sadkane • Ahmed Salam
}

Published online: 26 February 2014

(C) Springer Science+Business Media Dordrecht 2014

\section{The occasion}

This special issue of BIT contains papers related to talks given at the CIRM Workshop on Structured Matrix Computations in Non-Euclidean Geometries: Algorithms and Applications, held at the Centre International de Rencontres Mathématiques (CIRM) at the Luminy Campus of the Universite d'Aix-Marseille, October 8-12, 2012. The workshop was organized by the guest editors of this special issue, together with Lothar Reichel (Kent State University, USA). Its scientific committee (see Fig. 1) included the four main organizers as well as David Watkins (Washington State University, Pullman, USA) and Heike Faßbender (TU Braunschweig, Germany). The intention of the workshop was the cross-fertilization between researchers working on applications of non-Euclidean geometries, such as geometric integration or control theory, and people focusing on the analysis and numerics of structured matrices. Participants in the workshop are shown in Fig. 2 and a short report on the meeting can be found in

\footnotetext{
P. Benner $(\varangle)$

Max Planck Institute for Dynamics of Complex Technical Systems, Sandtorstr. 1,

39106 Magdeburg, Germany

e-mail: benner@mpi-magdeburg.mpg.de
}

M. Sadkane

CNRS, UMR 6205, Laboratoire de Mathématiques de Bretagne Atlantique,

Université de Brest, 6 Av. Le Gorgeu, 29238 Brest Cedex 3, France

e-mail: miloud.sadkane@univ-brest.fr

A. Salam

ULCO LMPA, Université Lille Nord de France, BP 699, 62228 Calais Cedex, France

e-mail: salam@1mpa.univ-littoral.fr 


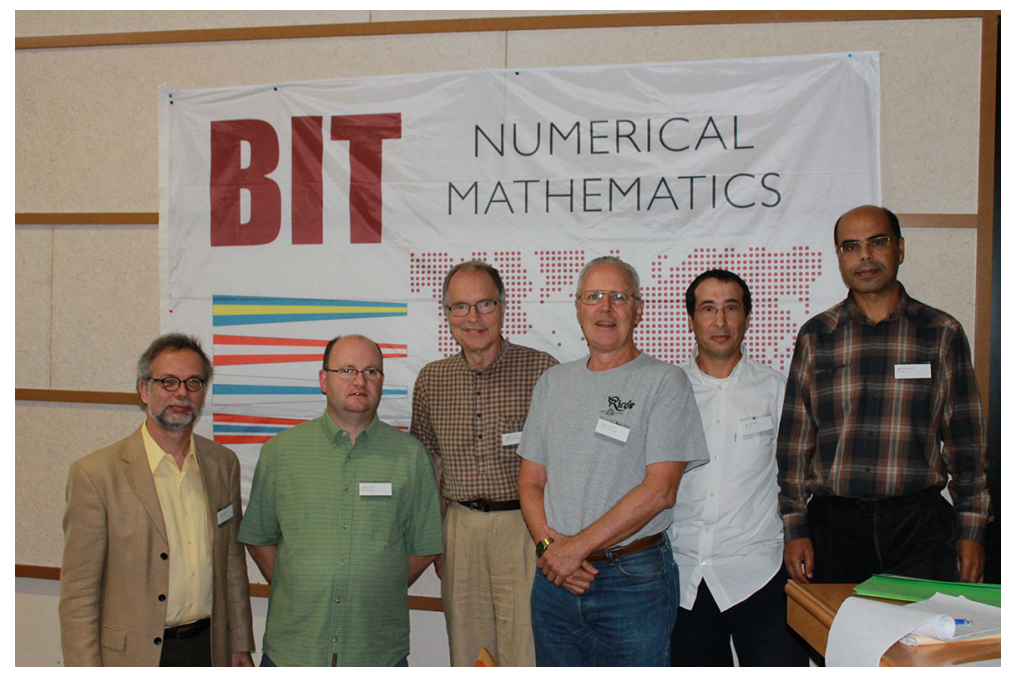

Fig. 1 The scientific committee of the workshop (without Heike Faßbender), together with the Editor-inChief of BIT (Axel Ruhe), during the workshop in Luminy. From left to right: Lothar Reichel, Peter Benner, Axel Ruhe, David Watkins, Ahmed Salam, Miloud Sadkane. Photo by courtesy of Stéphanie Vareilles/CIRM

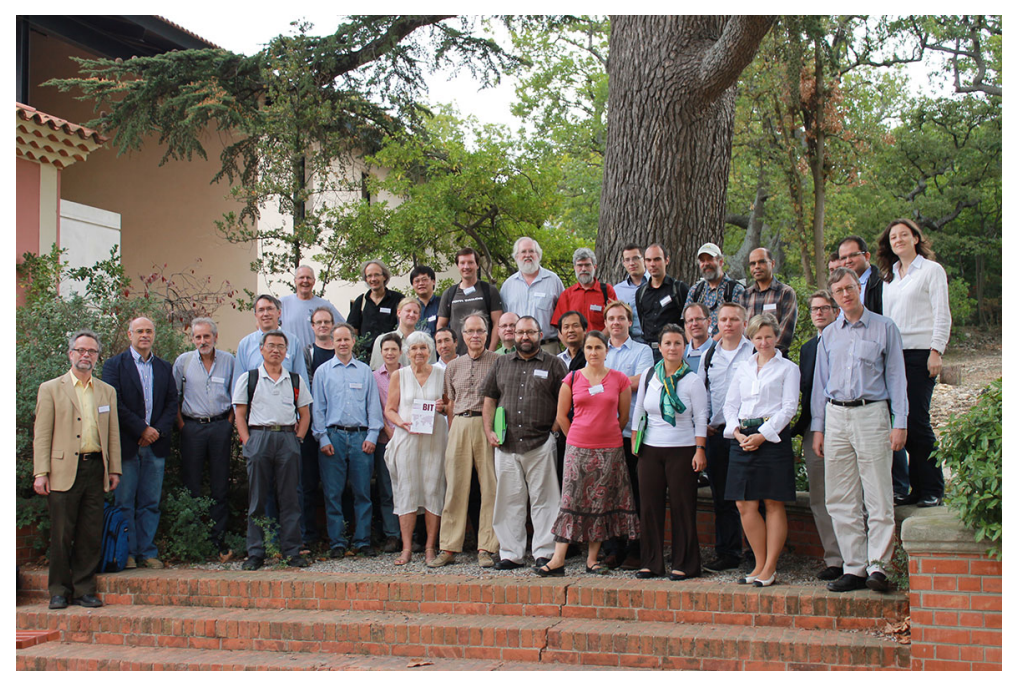

Fig. 2 The participants of the workshop. Photo by courtesy of Stéphanie Vareilles/CIRM

the Bulletin of the International Linear Algebra Society (ILAS), IMAGE, in volume 49, pp. $36-37^{1}$.

The main motivation in organizing a workshop at the intersection of Numerical Linear Algebra and Geometric Integration (and other application areas employing

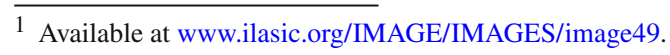


non-Euclidean geometries) was twofold. On the one hand side, in recent years, a lot of effort has been spent in the area of structured matrix computations. Besides the classical matrix structures like Toeplitz, Hankel, Cauchy, Vandermonde(-like), or circulant matrices, for which a rich body of efficient methods for matrix factorization and linear system solves now exists, another direction concerns matrices that arise as the adjoint and isometric operators in non-Euclidean geometries. This concerns for instance (skew-)Hamiltonian and symplectic matrices which are related to the linear symplectic vector space, or operators in (generalized) Minkowski space, i.e., operators that are (skew-)self-adjoint or isometric w.r.t. indefinite inner products induced by a diagonal metric tensor (signature matrix). A lot of this work was inspired by applications from control theory and concerned mostly problems of small to medium size so that dense matrix algebra could be used for computational methods to tackle eigenproblems or linear system solves. For large-scale, sparse problems, there are many open problems, and efficient algorithms that respect and employ the underlying structures are sought.

On the other hand and at the same time, challenging computational problems in the sciences and engineering have evolved that also require operations with structured matrices as the ones mentioned above. These include Hamiltonian mechanics and computational physics and chemistry problems like electronic structure calculations, where often long-time simulations are required. For these areas, geometric integration methods have been developed since the early 1990s, mostly without any knowledge of corresponding activities in Numerical Linear Algebra. As the need to solve larger and larger problems using more robust integrators arises, it becomes apparent that novel ideas from Numerical Linear Algebra are needed. A typical scenario is the integration of Hamiltonian systems, where modern techniques like exponential integrators require the application of matrix functions to structured matrices like the ones mentioned above.

Therefore, the aim of this workshop was to bring together researchers from Numerical Linear Algebra who have worked in structured matrix calculations with scientists in the application areas dealing with operators in non-Euclidean spaces. A particular emphasis was given to the area of geometric integrators, but also to other application fields like control theory and image processing.

During the meeting, we had the opportunity to congratulate Axel Ruhe, BIT's editor-in-chief, to his 70th birthday. One day of the meeting was devoted to this special occasion, and talks on this day were scheduled so as to link to Axel's research work.

The papers in this issue of BIT NUMERICAL MATHEMATICS reflect the interdisciplinary character of the workshop. A brief introduction to the papers contained in this volume is provided in the next section.

\section{The papers}

The 13 papers in this issue cover most of the workshop topics. Main topics in Numerical Linear Algebra for structured matrices are the preservation and exploitation of the structure in algorithms to compute eigenvalues and eigenvectors as well as the fast solution of linear systems. For eigenvalue problems, exploiting the structure may 
save a lot of computations and lead to faster algorithms. On the other hand, structure may impose symmetries in the spectrum that a numerical method should be able to reproduce which is not possible when using, e.g., the Francis QR algorithm in general. Several papers in this issue therefore treat structured eigenvalue problems. In "Minimization Principles and Computation for the Generalized Linear Response Eigenvalue Problem", Bai and Li show how a special Hamiltonian eigenproblem arising in linear response theory, a branch of computational physics, can efficiently be solved using a locally optimal block conjugate gradient-like algorithm by exploiting the special block structure of the matrices involved. General Hamiltonian eigenproblems are often treated by algorithms using symplectic transformations as they preserve the structure of Hamiltonian matrices. Such transformations can be obtained in various ways. Salam and Al-Aidarous show the "Equivalence between modified symplectic Gram-Schmidt and Householder SR algorithms" for this purpose. Symplectic structures and other matrix structures imposed from the automorphism groups associated to bilinear forms are even more difficult to treat numerically as they are given only implicitly. The "Eigenvalue perturbation theory of structured matrices under generic structured rank one perturbations: Symplectic, orthogonal, and unitary matrices" by Mehl, Mehrmann, Ran and Rodman provides theoretical insight into the behavior of the spectra of such matrices under perturbations of particular structure. Distance problems in matrix theory are another facet of perturbation theory as they allow to estimate the size of perturbations that are allowed to avoid possible singularities. In "A bisection method for measuring the distance of a quadratic matrix pencil to the quadratic matrix pencils that are singular on the unit circle", Malyshev and Sadkane treat a particular distance problem which is then solved using a structured eigenvalue algorithm.

A different type of structured eigenvalue problems is encountered when considering dense matrices with certain blocks that can be represented in a data-sparse fashion. Boito, Eidelman and Gemignani devise in their paper "Implicit QR for rank-structured matrix pencils" a modified QZ algorithm that computes the generalized eigenvalues of certain rank structured matrix pencils using $\mathcal{O}\left(n^{2}\right)$ flops and $\mathcal{O}(n)$ memory storage only. The same efficiency in computational and memory cost is obtained by the non-unitary analogue of Francis's implicitly-shifted QR algorithm for Hessenberg matrices of banded-plus-spike form described in "Fast computation of eigenvalues of companion, comrade, and related matrices" by Aurentz, Vandebril, and Watkins.

"Refining Estimates of Invariant and Deflating Subspaces for Large and Sparse Matrices and Pencils" is the topic of the paper by Fan, Weng, Chu and Lin. The suggested method uses algebraic Riccati equations, a type of quadratic matrix equation associated with Hamiltonian-like ${ }^{2}$ structures.

Exploiting particular matrix structures in the fast solution of overdetermined and ill-posed linear systems is the topic of "An algorithm for solving the indefinite least squares problem with equality constraints" by Mastronardi and Van Dooren, where a numerically forward stable reduction to anti-triangular form is introduced, as well as of "The structure of iterative methods for symmetric linear discrete ill-posed problems"

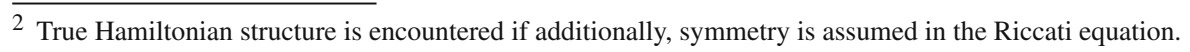


by Dykes, Marcellan and Reichel who investigate the structure of the matrices arising in range restricted minimal residual methods for large linear discrete ill-posed problems with a symmetric matrix.

Three papers in this issue address geometric and exponential integrators and related topics. Munthe-Kaas, Quispel and Zanna show in "Symmetric spaces and Lie triple systems in numerical analysis of differential equations" that a large variety of different numerical algorithms can be understood and analyzed using the concepts from differential non-Euclidean geometry. These considerations also lead to new insight into and improvements of self-adjoint numerical integration schemes. In "Comparison of methods for computing the action of the matrix exponential", Caliari, Kandolf, Ostermann and Rainer describe a new implementation of an exponential integration method based on interpolation at Leja points and numerically compare this method with others from the literature. Low-rank structures as discussed in some of the papers related to eigenproblems also often appear in time-dependent problems. Tracking a low-rank factor of a matrix-valued function in time is the topic of "A projector-splitting integrator for dynamical low-rank approximation" by Lubich and Oseledets.

Another topic covered in this issue is treated in the paper "Geometric means of structured matrices" by Bini, Iannazzo, Jeuris and Vandebril, where a new definition of a geometric mean for structured matrices is introduced, allowing the structurepreserving computation of the average of an ensemble of matrices. 